\title{
IKAROS Extended Operation for Advanced Solar Power Sail Mission
}

\author{
By Osamu Mori ${ }^{1}$, Ryu Funase ${ }^{2)}$, Yoji ShIRASAwA ${ }^{1)}$, Yuya MiMASU ${ }^{1)}$, Yuichi TsudA ${ }^{1)}$, Takanao SAIKI ${ }^{1)}$, \\ Hajime YANO $^{1)}$, Shuji MATSUURA ${ }^{1)}$, Daisuke YONETOKU ${ }^{3)}$ and Junichiro KAWAGUCHI ${ }^{1)}$ \\ 1) Japan Aerospace Exploration Agency, Sagamihara, Japan \\ ${ }^{2)}$ Department of Aeronautics and Astronautics, The University of Tokyo, Tokyo, Japan \\ ${ }^{3}$ School of Mathematics and Physics, Kanazawa University, Kanazawa, Japan
}

(Received June 18th, 2013)

\begin{abstract}
This paper presents the extended missions of the IKAROS solar sail interplanetary spacecraft. IKAROS entered an extended operation phase at the beginning of 2011. During this phase, its spin rate was reduced to observe deformation of the sail membrane under low centrifugal forces, and on Oct. 18, 2011 the spin was reversed to enhance knowledge of the membrane's stiffness against solar radiation pressure. We also investigated changes in IKAROS's attitude motions under reverse spin conditions. At the end of 2011, IKAROS switched to hibernation mode due to an increased Sun angle reducing on-board electricity generation. We later searched for IKAROS considering its attitude and orbital motion during hibernation, and on Sep. 6, 2012, we succeeded in locating the spacecraft, which came out of hibernation.

A solar power sail can be part of a hybrid propulsion system, with electrical power generated by thin-film solar cells on a solar sail membrane being used to operate ultra-high specific impulse ion engines. This paper also introduces a follow-on mission to IKAROS, a round trip to the Trojan asteroid using hybrid electric photon propulsion.
\end{abstract}

Key Words: Solar Power Sail, IKAROS, Extended Operation, Trojan Asteroid, Round Trip

\section{Introduction}

A Solar Sail is a space yacht that uses the pressure of sunlight on a large membrane for propulsion. It thus can move forward without consuming propellant as long as it can harness enough energy from sunlight. A Solar Power Sail is an original Japanese concept in which electrical power is additionally generated by thin-film solar cells on the sail membrane. This power can be used for electrical propulsion to supplement the thrust from photon pressure.

The Japan Aerospace Exploration Agency (JAXA) achieved the world's first demonstration of a solar power sail on the IKAROS (Interplanetary Kite-craft Accelerated by Radiation Of the Sun) spacecraft ${ }^{1)}$ during interplanetary cruise. IKAROS was launched in May 2010 and achieved all its primary mission goals in seven months. It then entered an extended operation phase at the beginning of 2011.

There are two types of solar sail. The first is a mast type en $^{2,3)}$ which uses a rigid support structure to deploy and maintain the sail. The other is a spin type ${ }^{4)}$ which uses the centrifugal force generated by spinning. A spin-type sail can be realized with lighter mechanisms than the mast type because it does not require rigid structural elements. IKAROS has a spin-type solar sail.

Because the stiffness of the sail membrane is very low, during normal operations the spacecraft's rate of spin is maintained at greater than $1 \mathrm{rpm}$ to keep the sail flat. During the extended operation phase, however, experiments were carried out to understand the behavior of the sail and the spacecraft under different spin conditions. The spin rate was first reduced to observe the deformation of the sail under the lower centrifugal force. Then, on Oct. 18, 2011, IKAROS transferred to reverse spin to enhance knowledge about the membrane stiffness against solar radiation pressure.

The attitude motion of a spinning solar sail is governed by solar radiation pressure. Viewed in Sun-fixed coordinates, the spin axis describes a spiral motion centered on a certain equilibrium point (spiral behavior). The spin rate varies linearly (windmill behavior). We therefore also investigated changes of IKAROS's attitude motion during the reverse spin mission.

At the end of 2011, IKAROS switched to hibernation mode because an increase in the Sun angle reduced on-board electrical generation. We later searched for IKAROS considering its attitude and orbital motion during hibernation, and on Sep. 6, 2012, we succeeded in locating the spacecraft, which came out of hibernation.

A solar power sail spacecraft can not only use a solar sail to save propellant but can also generate electrical power using a vast area of thin-film solar cells on the sail membrane, even at large distances from the Sun. By using the electrical power to operate ultra-high specific impulse ion engines, a hybrid propulsion system can be realized. A solar power sail is applicable to missions to the outer planetary region. As a follow-on to IKAROS, we propose a round trip mission to the Trojan asteroid using the solar power sail as shown in Fig. 2.

In this paper, the IKAROS missions during extended operations are presented, and the advanced solar power sail mission is introduced. 


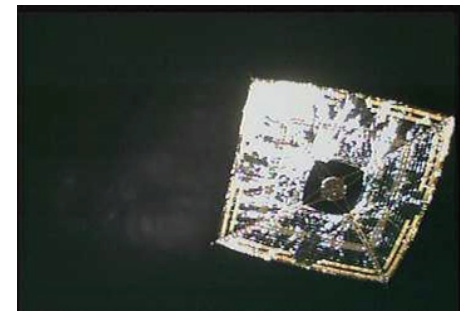

Fig. 1. IKAROS mission.

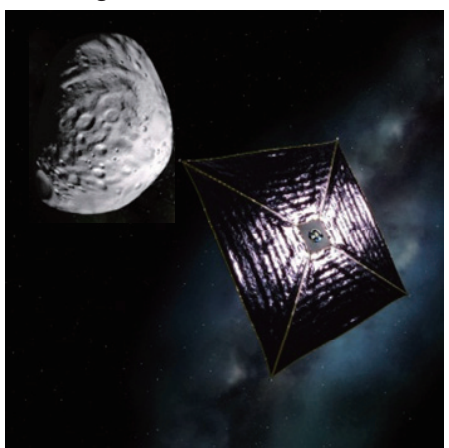

Fig. 2. Advanced solar power sail mission.

\section{IKAROS Mission}

\subsection{Initial and normal operations}

IKAROS's primary mission had the following two minimum success criteria:

(1) Deployment of a large sail membrane

(2) Generation of electricity by thin-film solar cells and the following two full success criteria:

(3) Demonstration of photon propulsion

(4) Demonstration of guidance, navigation and control techniques for solar sail propulsion

IKAROS was launched together with the Venus Climate Orbiter, AKATSUKI, on May 21, 2010. The planned mission sequence is shown in Fig. 3. After spin separation from the launch vehicle, IKAROS spun up to $25 \mathrm{rpm}$ and deployed its sail using centrifugal force. Power generation by thin-film solar cells was confirmed after deployment. It took several weeks to achieve the minimum success criteria during the initial operation phase. Photon propulsion was then verified and guidance, navigation and control using the solar sail were demonstrated, achieving full mission success during the normal operation phase. IKAROS flew by Venus on Dec. 8, 2010.

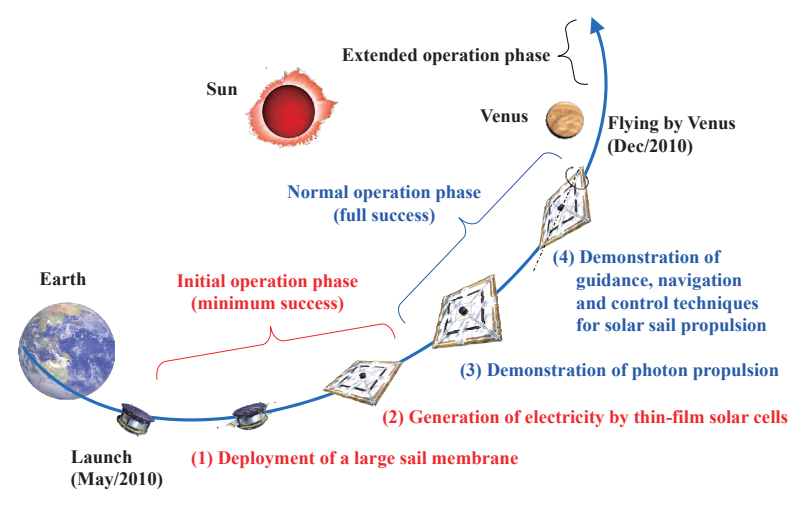

Fig. 3. IKAROS mission sequence.

\subsection{Extended operation - slow spin mission}

To expand and maintain the shape of the sail membrane against solar radiation pressure, the spin rate of IKAROS was maintained at greater than $1 \mathrm{rpm}$ for about one year from the completion of sail deployment on June 9, 2010.

After the primary mission goals were successfully achieved, IKAROS entered an extended operation phase during which experiments were carried out to explore different spin conditions. A slow spin experiment was started in June 2011 to observe the membrane stiffness against solar radiation pressure under low centrifugal force.

The spin rate of IKAROS is naturally reduced by solar radiation pressure torque (windmill behavior). During the normal operation phase, the spin rate had been maintained by periodical thrust control (gas jet injection). By suspending this spin control, the spin rate reduced to $0.055 \mathrm{rpm}$ in the slow spin mission.

Figure 4 shows the angular velocity of the main body at $0.055 \mathrm{rpm} . \omega_{z}$ indicates the angular velocity around the $z$-axis, which is equivalent to the spin rate shown in Fig. 5. This indicates that the oscillations of out of plane angular velocities $\omega_{x}$ and $\omega_{y}$ are nearly equal to the spin rate. This nutation motion can arise when the inertia tensor of the system is close to that of a thin plate. From this result, it is considered that the main body of the spacecraft had rotated along with the sail membrane.

Images of the sail were taken by side monitor cameras. Figure 6 shows sail images and the sail shapes predicted by numerical simulations ${ }^{5)}$ at $2.5 \mathrm{rpm}$ and $0.055 \mathrm{rpm}$. Table 1 shows the out-of-plane displacement at the corner of the sail predicted by the simulations and estimated from the camera images. Although it had been expected that the decrease of centrifugal force would cause deformation of sail by solar radiation pressure, the camera images show that the sail shape at $0.055 \mathrm{rpm}$ is not very different from that at $2.5 \mathrm{rpm}$.

From these results, we concluded that the sail membrane has a greater bending stiffness on average than expected. Reflecting these findings, we modified the numerical models of the sail membrane used to simulate flight data.

After the slow spin mission, we planned a reverse spin mission. The sequence of thrust injections to achieve reverse spin was designed based on the result of simulations using the modified higher bending stiffness model.

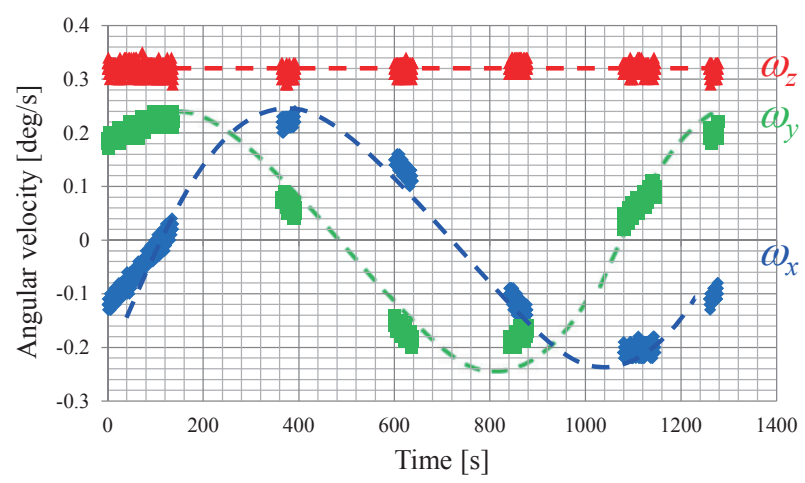

Fig. 4. Angular velocity of the main body during slow spin mission. 


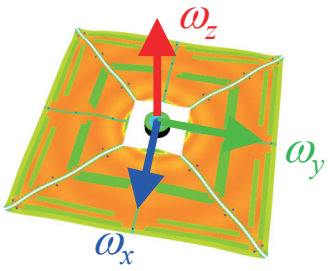

Fig. 5. Definition of body coordinate system.

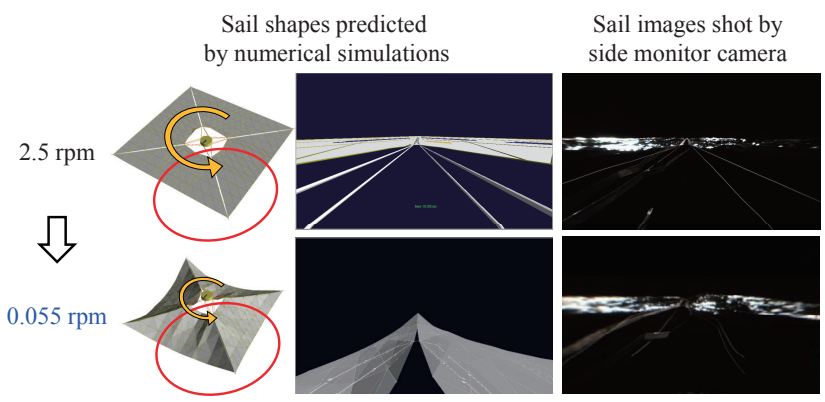

Fig. 6. Sail images and the sail shapes predicted by numerical simulations at $2.5 \mathrm{rpm}$ and at $0.055 \mathrm{rpm}$.

Table 1. Out-of-plane displacement at the corner of the sail

\begin{tabular}{ccccc}
\hline & \multicolumn{4}{c}{ Displacement [m] } \\
\cline { 2 - 5 } & \multicolumn{3}{c}{ Prediction by Simulations } & \multicolumn{2}{c}{ Estimation from Images } \\
\cline { 2 - 5 } & $2.5 \mathrm{rpm}$ & $0.055 \mathrm{rpm}$ & $2.5 \mathrm{rpm}$ & $0.055 \mathrm{rpm}$ \\
\hline CAM-H1 & 0.00 & -1.65 & -0.17 & -0.17 \\
\hline CAM-H2 & 0.00 & -1.72 & 0.21 & -0.54 \\
\hline CAM-H3 & 0.00 & -1.70 & -0.04 & 0.34 \\
\hline CAM-H4 & 0.00 & --1.63 & -0.07 & -0.07 \\
\hline
\end{tabular}

\subsection{Extended operation - reverse spin mission}

On Oct. 18, 2011, the reverse spin mission was performed to enhance knowledge about the stiffness of the sail membrane against solar radiation pressure and to investigate the spacecraft's attitude dynamics under the different spin direction.

The spacecraft's spin was reversed using gas jet thrusters with a pre-planned firing schedule. Thrust injections of $0.4 \mathrm{sec}$ pulse width were made 118 times at $10-$ second intervals. As a result, the spin rate changed from $0.15 \mathrm{rpm}$ to $-0.24 \mathrm{rpm}$, and the sail membrane was successfully spun in the reverse direction without becoming entangled.

Figure 7 shows the angular velocity of the main body during the reverse spin mission. The figure shows how the spin rate of the main body is reversed by the thrust injections. The spin rate change of the main body is reduced after the thrust injections by in-plane oscillations between the body and the sail membrane, which has a high moment of inertia; however it gradually shifts to the reverse direction.

Figures 8 and 9 show the sail images shot by the side monitor cameras at $0 \mathrm{rpm}$. These reveal that the shape of the sail was not greatly deformed even at $0 \mathrm{rpm}$. From these results, it is considered that the sail membrane of IKAROS has high bending stiffness, confirming the result of the slow spin mission.

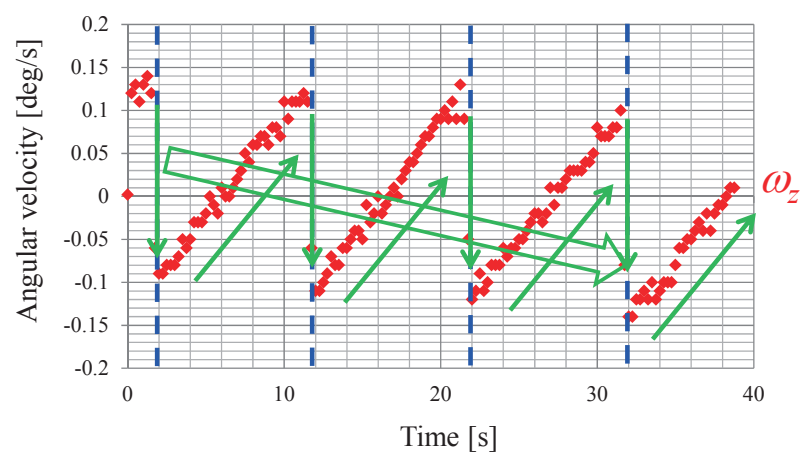

Fig. 7. Angular velocity of the main body during reverse spin mission.

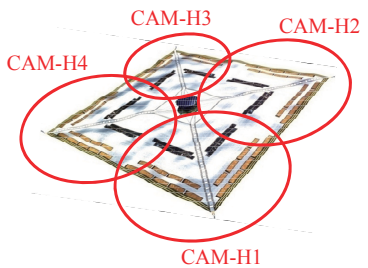

Fig. 8. Field of view of side monitor cameras.

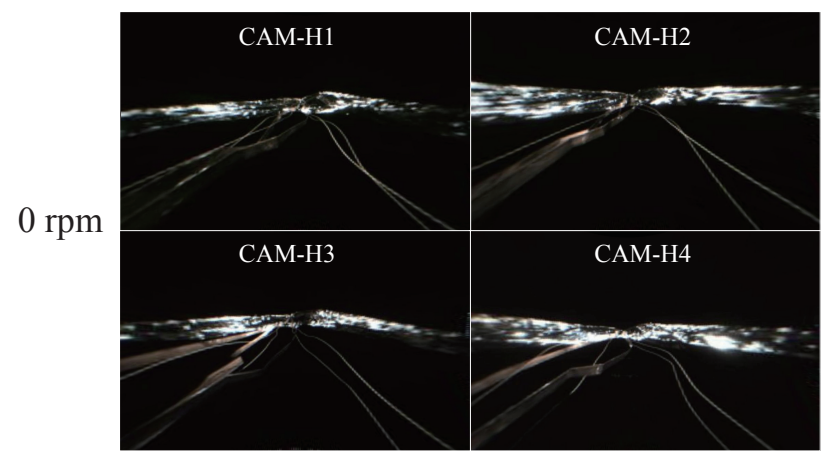

Fig. 9. Sail images at $0 \mathrm{rpm}$.

\subsection{Extended operation - attitude motion}

The general form of the spin-averaged dynamics of a spinning solar sail ${ }^{6)}$ is as follows:

$$
\frac{d}{d t}\left[\begin{array}{l}
\alpha \\
\delta \\
\Omega
\end{array}\right]=\left[\begin{array}{ccc}
\frac{1}{I_{S} \Omega} A & -\frac{1}{I_{S} \Omega} B & 0 \\
\frac{1}{I_{S} \Omega} B & \frac{1}{I_{S} \Omega} A & 0 \\
0 & 0 & \frac{1}{I_{S}} C
\end{array}\right]\left[\begin{array}{c}
\cos \left(\delta-\delta_{s}\right) \sin \left(\alpha-\alpha_{s}\right) \\
\sin \left(\delta-\delta_{s}\right) \\
1
\end{array}\right]
$$

where $\alpha, \delta$ are right ascension and declination of the spin vector with respect to the inertial frame, respectively, $\Omega$ is the spin rate, $\alpha_{s}, \delta_{s}$ are right ascension and declination of the Sun direction viewed from the spacecraft, respectively, and $I_{S}$ is the moment of inertia about the spin axis. $A, B$ and $C$ are torque parameters determined by the optical properties and the shape of the sail.

By assuming that the spin vector direction is very close to the Sun direction and the celestial equator ( $\left.\alpha-\alpha_{s}, \delta-\delta_{s}<<1\right)$, Equqtion (1) can be approximated as the following form: 


$$
\frac{d}{d t}\left[\begin{array}{l}
\alpha \\
\delta \\
\Omega
\end{array}\right] \approx\left[\begin{array}{ccc}
\frac{1}{I_{S} \Omega} A & -\frac{1}{I_{S} \Omega} B & 0 \\
\frac{1}{I_{S} \Omega} B & \frac{1}{I_{S} \Omega} A & 0 \\
0 & 0 & \frac{1}{I_{S}} C
\end{array}\right]\left[\begin{array}{c}
\alpha-\alpha_{S} \\
\delta-\delta_{S} \\
1
\end{array}\right]
$$

With this approximation we can rewrite $A, B$ and $C$ as functions only of the sail's optical properties and shape, eliminating their dependence on the attitude parameters (Sun angle).

Equation (2) can be solved when the Sun's apparent motion is very slow compared with the spin axis motion, and the spin rate variation is sufficiently slow $\left(\dot{\alpha}>>\dot{\alpha}_{s}, \dot{\delta}>>\dot{\delta}_{s}\right.$, $\Omega<<1)$. The solution is given by

$$
\begin{aligned}
& \alpha \approx \alpha_{e q}+D \exp \left(\frac{1}{I_{S} \Omega} A t\right) \cos \left(\frac{1}{I_{S} \Omega} B t+\varphi\right), \\
& \delta \approx \delta_{e q}+D \exp \left(\frac{1}{I_{S} \Omega} A t\right) \sin \left(\frac{1}{I_{S} \Omega} B t+\varphi\right), \\
& \Omega \approx \Omega_{0}+\frac{1}{I_{S}} C t
\end{aligned}
$$

where $D, \varphi$ and $\Omega_{0}$ are determined by initial conditions and

$$
\left[\begin{array}{c}
\alpha_{e q} \\
\delta_{e q}
\end{array}\right] \approx\left[\begin{array}{c}
\alpha_{s} \\
\delta_{s}
\end{array}\right]+\frac{I_{S} \Omega}{A^{2}+B^{2}}\left[\begin{array}{cc}
A & B \\
-B & A
\end{array}\right]\left[\begin{array}{c}
\dot{\alpha}_{s} \\
\dot{\delta}_{s}
\end{array}\right]
$$

Equation (3) shows that the spin axis tends to describe circles with an angular velocity of $B /\left(I_{S} \Omega\right)$ about an equilibrium point $\left(\alpha_{e q}, \delta_{e q}\right)$, and that the circling radius varies exponentially with the parameter $A /\left(I_{S} \Omega\right)$. The spin rate varies linearly with the parameter $C / I_{S}$. These behaviors were actually observed for IKAROS; the behavior of the spin axis is understood as spiral behavior, while the behavior of the spin rate can be understood as windmill behavior.

Figure 10 shows the attitude motion of IKAROS in the case of forward spin. The spin axis describes a clockwise spiral motion and converges at the equilibrium point $(A<0, B>0)$. The spin rate decreases linearly $(C<0)$. The equilibrium point is nearly equal to Sun direction point because the spin rate is sufficiently small.

Figure 11 shows the attitude motion in the case of reverse spin. The spin axis describes a counter-clockwise spiral motion and diverges from the equilibrium point $(A<0, B>0)$. The spin rate decreases and converges at -5.3 $\operatorname{rpm}(C<0)$.

The signs of $A, B$ and $C$ did not change during both the normal and extended operations, including the slow spin and reverse spin missions. This means that the optical properties and sail shape did not change dynamically after sail deployment. The spin direction tended to be inertia oriented according to the increase of absolute value of spin rate.

Because an increased Sun angle resulted in the electrical power generation rate becoming less than the power consumption, IKAROS switched to hibernation mode (shutdown) at the end of 2011.

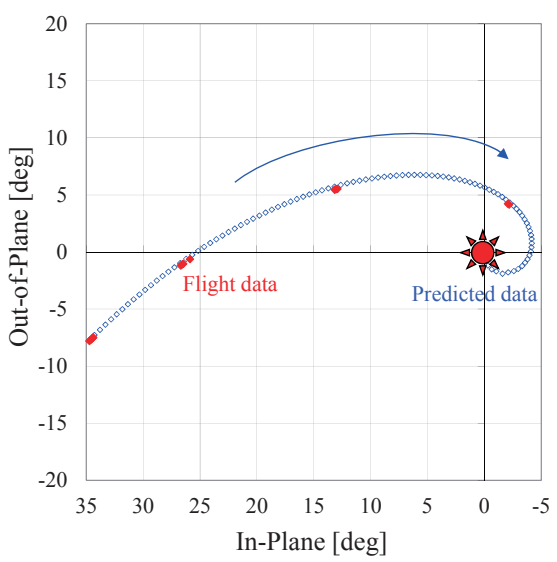

(a) Spin axis history between Sep. 29 and Oct. 11, 2011

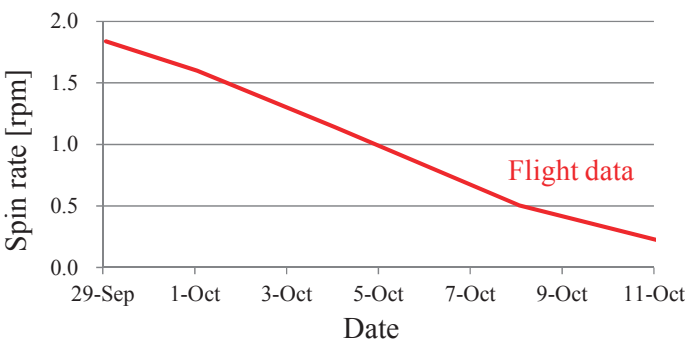

(b) Spin rate history between Sep. 29 and Oct. 11, 2011

Fig. 10. Attitude motion in the case of forward spin.

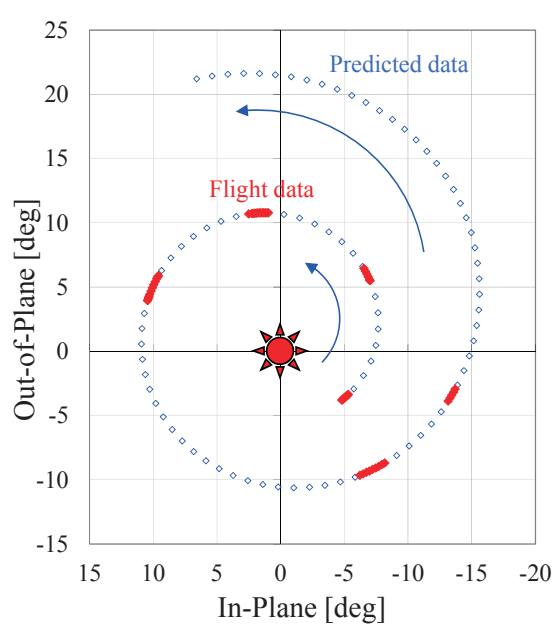

(a) Spin axis history between Sep. 29 and Oct. 11, 2011

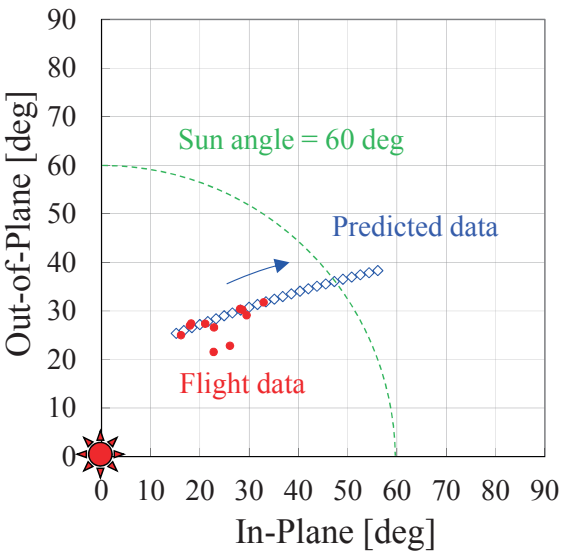

(b) Spin rate history between Sep. 29 and Oct. 11, 2011 
Date

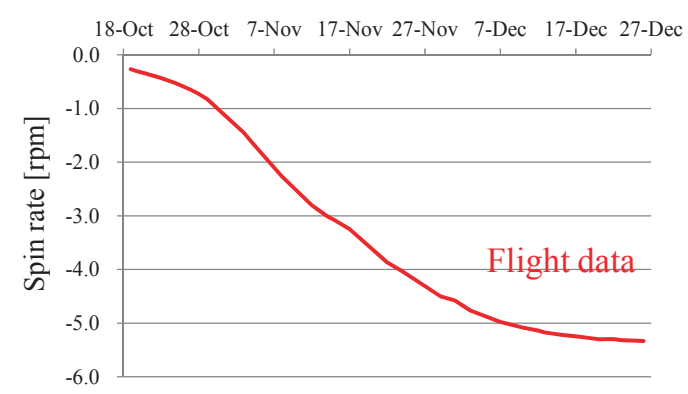

(c) Spin rate history between Oct. 18 and Dec. 26, 2011

Fig. 11. Attitude motion in the case of reverse spin.

\subsection{Extended operation - search mission}

Because IKAROS is accelerated by a solar sail, in order to search for it after hibernation we needed to calculate its orbit considering its attitude motion in detail. Fig. 12 shows the predicted attitude motion after switching to hibernation. The detail of this analysis is written in Reference 7). We succeeded in locating IKAROS on Sep. 6, 2012. IKAROS came out of hibernation in the latter half of September, 2012, as forecast from the predicted attitude motion.

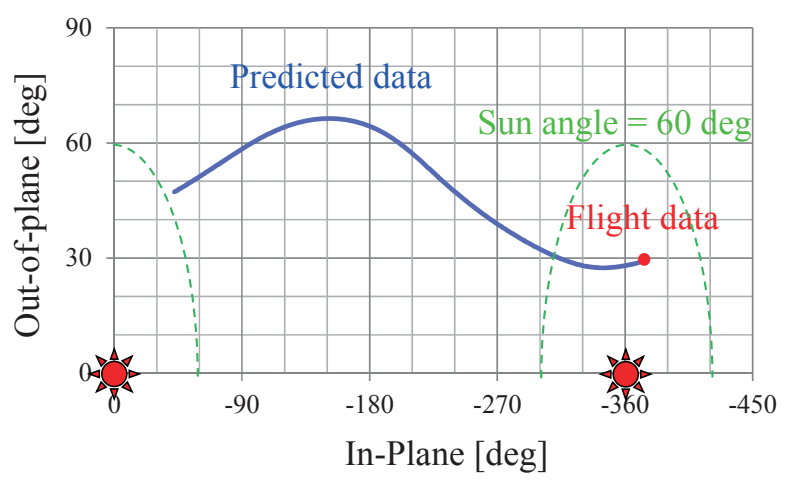

(a) Spin axis history between Dec. 24, 2011 and Sep. 6, 2012

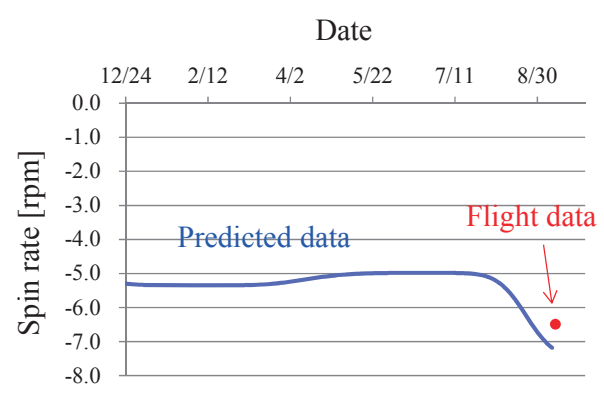

(b) Spin rate history between Dec. 24, 2011 and Sep. 6, 2012

Fig. 12. Attitude motion after switching to hibernation.

\section{Advanced Solar Power Sail Mission}

As a follow-on to the IKAROS mission, an advanced solar power sail craft is being designed as shown in Fig. 13. The area of the sail is $3000 \mathrm{~m}^{2}$, which is 15 times larger than that of IKAROS. The spacecraft can generate large electric power even in the outer areas of the solar system (7kW@5.2AU) using thin-film solar cells attached to the entire surface of the sail membrane. It will use the world's first hybrid photon / ion propulsion system. The specific impulse of the ion engine is
$6000 \sim 9000 \mathrm{sec}$, which is $2 \sim 3$ times larger than that of the Hayabusa asteroid probe.

Figure 14 shows the mission sequence of advanced solar power sail. The spacecraft will make a world's first round trip to the Trojan asteroid using Earth and Jupiter gravity assist with the following schedule.

2023: Launch

2025: Earth Swing-by

2029: Jupiter Swing-by

2037 2039: Trojan asteroid rendezvous / sampling

2047: Jupiter Swing-by

2049: Return to Earth

In addition, this innovative advanced solar power sail craft will carry the following demonstrations of new technologies that will be required for future solar system exploration:

1. A large membrane space structure including deployment strategy,

2. Thin-film solar cells,

3. Reflectivity control device,

4. Hybrid propulsion using both solar sail and ion engines,

5. Ultra-high specific impulse ion engines,

6. Reaction control system capable of operation at very low temperatures,

7. An integrated propulsion / power system using fuel cells.

8. Membrane phased-array antenna,

9. Ultra-stable oscillator for one-way ranging or VLBI orbit determination,

10. Radiation-resistant technology for Jovian Orbiter,

11. Reentry capsule

Technologies 1-4 have been partially demonstrated by IKAROS.

In addition to these technology demonstrations, the mission has several new innovative first-class planetary science and space physics objectives, as shown in Fig. 14. The spacecraft will perform infrared astronomy observations together with ecliptic dust detection during cruise, will make magnetosphere observations of Jupiter, and visit the Trojan asteroids.

The major characteristics of the advanced solar power sail mission are now summarized. It will feature the

1. World's First Photon / Electric Hybrid Sail Propulsion,

2. World's Highest Performance Ion Engines,

3. World's First Round Trip to the Outer Solar System

4. World's Highest Speed Reentry Capsule

5. World's First Background Emission Mapping,

6. World's First Access to Trojan Asteroids,

7. World's First Sample Return from Trojan Asteroid,

8. World's First Dust Return from Outer Solar System

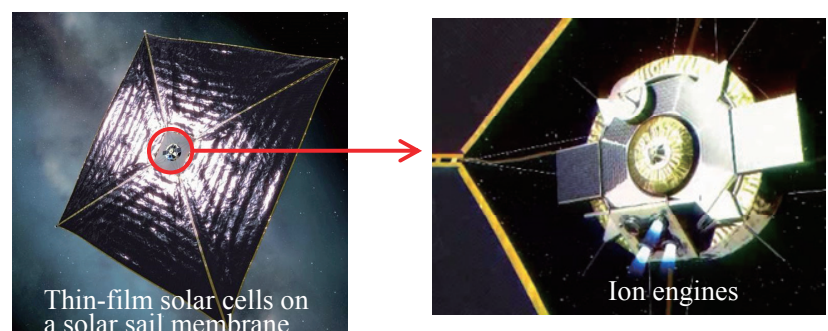

Fig. 13. Advanced solar power sail craft system. 


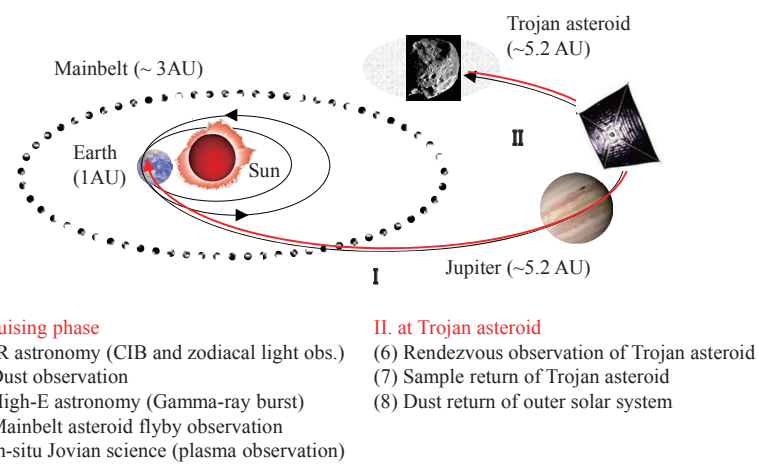

Fig. 14. Mission sequence of advanced solar power sail.

\section{Conclusions}

This paper presented experimental missions carried out during the extended operation phase of the IKAROS solar sail spacecraft.

In the slow spin mission, the shape of the sail did not greatly change from the normal spin rate shape. The sail membrane had a larger bending stiffness on average than expected. The main body became unstable by solar radiation pressure torque. This is also associated with the decrease of rotation stiffness.

In the reverse spin mission, the sail membrane's spin was successfully reversed without the sail becoming entangled. It is considered that the IKAROS sail membrane has large bending stiffness, confirming the finding of the slow spin mission.

The attitude motion using a generalized spinning sail model was shown. The behavior of the spin direction is understood as spiral behavior and the behavior of the spin rate is understood as windmill behavior. The signs of $A, B$ and $C$ did not change during normal and extended operations, including the slow spin and reverse spin missions. This indicates that the optical properties and shape of the sail did not change dynamically after deployment.

IKAROS switched to hibernation mode because the Sun angle increased. We searched for IKAROS considering its predicted attitude and orbital motion during hibernation, and succeeded in locating IKAROS which then came out of hibernation.

This paper also introduced plans for an advanced solar power sail mission including a round trip to the Trojan asteroid. It will use the world's first hybrid photon / ion propulsion system, taking advantage of thin-film photo-voltaic technology. Solar power sails are suitable for outer planetary exploration.

\section{References}

1) Mori, O., Tsuda, Y., Sawada, H., Funase, R., Yamamoto, T., Saiki, T., Yonekura, K., Hoshino, H., Minamino, H., Endo, T. and J. Kawaguchi: World's First Mission of Solar Power Sail by IKAROS, International Conference on Space, Aeronautical and Navigational Electronics, 2010, SANE2010-95.

2) Greschik, G. and Mikulas, M. M.: Design Study of a Square Solar Sail Architecture, J. of Spacecraft and Rockets, 39 (2002), pp.653-661.

3) Hinkle, J. D., Warren, P. and Peterson, L. D.: Geometric Imperfection Effects in an Elastically Deployable Isogrid Column, $J$. of Spacecraft and Rockets, 39 (2002), pp.662-668.

4) Sawada, H., Mori, O., Okuizumi, N., Shirasawa, Y., Miyazaki, Y., Natori, M., Matunaga, S., Furuya, H. and Sakamoto, H.: Mission Report on The Solar Power Sail Deployment Demonstration of IKAROS, 12th AIAA Gossamer Systems Forum, 2011, AIAA-2011-1887.

5) Shirasawa, Y., Mori, O., Miyazaki, Y., Sakamoto, H., Hasome, M., Okuizumi, N., Sawada, H., Matunaga, S., Furuya, H. and Kawaguchi, J.: Evaluation of Membrane Dynamics of IKAROS Based on Flight Result and Simulation Using Multi-Particle Model, 28th International Symposium on Space Technology and Science, 2011, 2011-0-4-05v.

6) Tsuda, Y., Saiki, T., Mimasu, Y. and Funase, R.: Modeling of Attitude Dynamics for IKAROS Solar Sail Demonstrator, AAS/AIAA Space Flight Mechanics Meeting, 2011, AAS11-112.

7) Mimasu, Y., Taniguchi, S., Takeuchi, H., Shirasawa, Y., Yonekura, K., Mori, O., Funase, R., Saiki, T. and Tsuda, Y., "Long-term Attitude and Orbit Prediction of Solar Sailing IKAROS While Being Lost in Space," The 23rd AAS/AIAA Space Flight Mechanics Meeting, 2012, AAS13-406. 